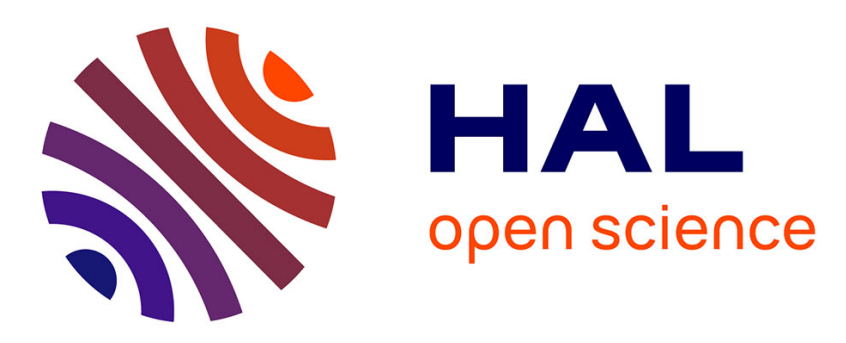

\title{
Stability of two-layer shear-thinning film flows
}

Séverine Millet, Valéry Botton, Hamda Ben Hadid, Daniel Henry, François

Rousset

\section{To cite this version:}

Séverine Millet, Valéry Botton, Hamda Ben Hadid, Daniel Henry, François Rousset. Stability of twolayer shear-thinning film flows. Physical Review E: Statistical, Nonlinear, and Soft Matter Physics, 2013, 88 (4), pp.043004. 10.1103/PhysRevE.88.043004 . hal-00992095

\section{HAL Id: hal-00992095 \\ https://hal.science/hal-00992095}

Submitted on 11 Apr 2016

HAL is a multi-disciplinary open access archive for the deposit and dissemination of scientific research documents, whether they are published or not. The documents may come from teaching and research institutions in France or abroad, or from public or private research centers.
L'archive ouverte pluridisciplinaire HAL, est destinée au dépôt et à la diffusion de documents scientifiques de niveau recherche, publiés ou non, émanant des établissements d'enseignement et de recherche français ou étrangers, des laboratoires publics ou privés. 


\title{
Stability of two-layer shear-thinning film flows
}

\author{
S. Millet, V. Botton, H. Ben Hadid, and D. Henry \\ Laboratoire de Mécanique des Fluides et d'Acoustique, UMR-CNRS 5509, Université de Lyon, \\ École Centrale de Lyon/Université Lyon I/INSA-Lyon, 69622 Villeurbanne Cedex, France
}

\section{F. Rousset}

Université de Lyon, CNRS, INSA-Lyon, CETHIL, UMR5008, F-69621, Villeurbanne, France and Université Lyon 1, F-69622, France

(Received 16 April 2013; revised manuscript received 23 September 2013; published 14 October 2013)

\begin{abstract}
The stability of a two-layer film flow of non-Newtonian fluids is studied with a linear temporal approach. Shear-thinning fluids are considered, which follow the four-parameter inelastic Carreau model. A modified Orr-Sommerfeld equation system is obtained, which is solved by using a spectral Tau collocation method based on Chebyshev polynomials. The effects of density and viscosity stratification are considered, as well as the influence of the shear-thinning properties of the fluid. It is found that, when the viscosity is stronger in the upper layer, the base flow and the stability properties are almost not influenced by the change of the shear-thinning properties in this upper layer. In the other situations, the shear-thinning properties have an influence on the different instabilities, the long-wave surface instability and the short- and long-wave interface instabilities.
\end{abstract}

DOI: 10.1103/PhysRevE.88.043004

PACS number(s): 47.20.Ma, 47.50.Gj, 47.15.gm

\section{INTRODUCTION}

The hydrodynamics of layered film flows driven by gravity down an inclined plane is of practical interest in many chemical engineering applications, for example, in the processes involving the simultaneous coating of multiple layers, such as slide coating and curtain coating [1]. Geophysical phenomena such as glaciers [2,3], mud and lava flows [4] or granular flows [5] also imply this kind of configuration. Most of the studies in the literature on this topic are based on a Newtonian fluid model. The rheological behavior of many fluids cannot, however, be properly described by a Newtonian model, and more sophisticated rheological models involving a nonlinear relationship between stress and strain would be more appropriate. For example, most of the fluids used in the plastic manufacturing industry or in coating processes for paper or painting industries are basically shear thinning (colloids, suspensions, and a variety of polymeric liquids). This is also the case for glaciology [3], molten metals or lava, low molecular weight biological fluids, blood, etc. Note that many of those fluids have a shear-thinning behavior only for a certain range of shear rates, and they may also show yield stress behavior for low temperatures and/or high densities. In most of these applications, multilayer flows are involved.

The case of superposed layers of Newtonian fluid films flowing down an inclined plane has been extensively studied over the past few years, and it has been shown that these flows can easily become unstable due to waves traveling along the interfaces. A long-wave approximation is commonly adopted in the analytical approaches because the most dangerous instability is assumed to have a wavelength much larger than the layer thickness. This assumption was first used by Benjamin [6] and Yih [7], who considered a single layer of Newtonian fluid. They showed that the critical Reynolds number for the onset of the instabilities depends only on the inclination of the plate $\beta$ and is proportional to $\cot \beta$. They also

\footnotetext{
*severine.millet@univ-lyon1.fr
}

pointed out that inertia is required to trigger these free surface instabilities.

A similar conclusion is reached for confined Poiseuille or Couette multilayer flows [8,9] or for multilayer flows with an upper layer of infinite depth [10]: inertia is required to destabilize the liquid-liquid interface in these cases.

Kao [11-13], Loewenherz and Lawrence [2], and Chen [14] have studied the linear stability of a two-layer film flow with a free surface. With a zero Reynolds number approximation, they have shown that a new kind of instability could develop when the viscosity of the upper layer is greater than that of the lower layer. This instability was called inertialess instability. They also explained that, for multilayer flows, there are specific instability modes for each interface and free surface. Without inertia, the free surface mode is always stable, so that the instability can only be triggered by an interface mode. The stability curves showing the interfacial perturbation growth rate as a function of the wave number indicate that two modes with different wavelengths can become unstable: a first mode with a short wavelength, which is influenced by the layer thickness ratio, and a second mode with a long wavelength. This long-wave mode was already observed within the long-wave approach by Kao [11] and was associated to a so-called antilubrication effect. Among these short-wave and long-wave modes, the dominant mode, which will impose the instability wavelength, will be the mode with the larger growth rate. It was found that these growth rates depend on the surface tensions and the viscosity and thickness ratios, so that changes of dominant modes can occur. In the opposite configuration, where the more viscous layer is adjacent to the wall, no instability occurs, as far as inertia is not accounted for. These works have shown that, though the free surface is not responsible for the release of instabilities with respect to confined flows results, its presence plays an essential part in this process. The authors conclude that a strong interaction between surface and interface modes should exist.

Hu et al. $[15,16]$ extended these works on two-layer film flow down an incline by focusing on the effect of the density stratification and by using combined temporal, spatiotemporal, 
and spatial approaches. They considered both an inertialess configuration [15] and a configuration with inertia [16]. The decrease of the density ratio [(upper layer/lower layer)] always make the surface mode and the short-wave interface mode more stable. The short-wave interfacial instability can even disappear whatever the Reynolds number is, when the less viscous layer is in the region next to the wall. On the other hand, the decrease of the density ratio favors the long-wave interfacial instability. The transition between the long-wave and short-wave interfacial instabilities was also encountered in the spatiotemporal analysis. The authors have shown that these instabilities are generally convective and that the transition is associated with a jump in the local oscillatory frequency, spatial amplification rate, and spatial wave number.

Jiang et al. [17] performed an energy budget on the twolayer flow problem in order to explain these differences in stability according to whether the flow is confined or not and to the viscosity stratification. The authors have shown that a key role is played by the work of the shear stress at the undisturbed free surface, similarly to what was found in the one-layer free surface problem.

Besides these works which were all concerned by Newtonian fluids, some studies have considered non-Newtonian behaviors. Weinstein [18] numerically studied the influence of the Carreau constitutive equation on the spatial stability of a multiple-layer film flow down an inclined plane. In order to assess the effect of the shear-thinning behavior, the author compared the non-Newtonian results with the two limiting Newtonian cases based on the minimum and maximum viscosities attained in the shear-thinning layer. $\mathrm{He}$ observed that the shear-thinning results lie between these two Newtonian cases except at very low frequencies. The author finally proposed a mechanism based on energy considerations that models the different cases.

Balmforth et al. [3] explored the interfacial instability in the two-layer flow down an incline for power-law fluids. Due to zero Reynolds number expansions, they built simplified models to solve the linear stability problem and extended this work with weakly nonlinear analyses to explore the dynamics of the unstable waves. However, they met difficulties when the upper layer is non-Newtonian because of the constitutive law they chose: this power-law predicts an infinite viscosity for zero shear rate, which necessarily occurs at the free surface. Their difficulties to obtain consistent results in this configuration point out the limit of the power-law model for free surface flow investigations.

Moreover Usha et al. [19] have compared the stability threshold obtained with a Carreau model and a power-law model for a shear-thinning film on a rigid or porous substrate. They have shown that the choice of the model induces considerable changes on the stability, especially for weakly shear-thinning fluids.

Finally, Rousset et al. [20] studied the temporal stability of a Carreau fluid flow down an inclined plane. They performed an asymptotic approach considering a weakly non-Newtonian behavior in the limit of very long waves and compared it with a more general numerical approach. It was found that the critical Reynolds number is lower for shear-thinning fluids than for Newtonian fluids, while the wave celerity is larger. A particular attention was paid to the situations with small angles of inclination. Indeed, in these cases, besides the long-wave free surface mode, another instability identified by Floryan et al. [21] as a shear mode can occur. It is characterized by a wavelength on the order of the layer thickness and a wave celerity lower than the free surface velocity. It was shown that taking into account the shear dependence of the viscosity can change the nature of the instability.

In this paper we present the linear stability analysis of a two-layer non-Newtonian film flow driven by gravity down an incline. We focus on shear-thinning fluids (i.e., with a viscosity locally decreasing with the increase of the shear rate) and use the four-parameter Carreau inelastic model [22]. Such fluids correspond, for example, to mud suspensions, paints, or polymeric solutions. The model predicts a region at moderate shear rate in which the fluid follows a power-law behavior. However, unlike the power-law model, the Carreau model predicts a viscosity that remains constant as the shear rate approaches zero. This feature makes the Carreau law particularly suitable for free surface flow issues.

\section{FORMULATION}

\section{A. Base flow}

We study a two-layer laminar fluid flow driven by gravity down an inclined plane. The angle of inclination to the horizontal is called $\beta$. The two liquid layers are considered immiscible. Subscript $k=1,2$ denotes the upper and lower layer, respectively, as shown in Fig. 1, and the overbar denotes dimensional quantities. These conventions are adopted for all the fluid properties. Thus $\bar{d}_{k}$ is the thickness of layer $k, \bar{\rho}_{k}$ is its density, and $\bar{\eta}_{k}$ is its dynamical viscosity. The surface tension at the free surface between the upper layer and the passive gas above it is denoted as $\bar{\sigma}_{1}$, whereas $\bar{\sigma}_{2}$ stands for the surface tension at the interface between the two layers. The flow rate per unit width in each layer, $\bar{Q}_{k}$, is also specified, defining the total flow rate per unit width, $\bar{Q}_{T}=\bar{Q}_{1}+\bar{Q}_{2}$. We use a Cartesian coordinate system in which the $\bar{x}$ axis is aligned with the inclined plane and points down the slope and the $\bar{y}$ axis is taken normal to the plane and oriented towards it. The origin is taken at the undisturbed free surface. $\bar{\zeta}_{1}(\bar{x}, \bar{t})$ is the free surface location, and $\bar{\zeta}_{2}(\bar{x}, \bar{t})$ is the interface location. The governing

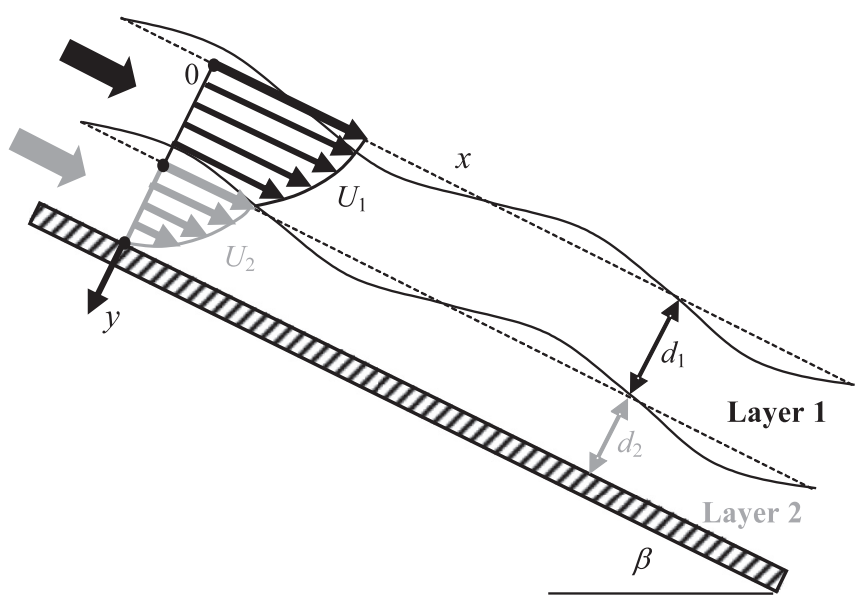

FIG. 1. Definition sketch of the dimensionless two-layer system. 
continuity and Navier-Stokes equations are

$$
\begin{gathered}
\frac{\partial \bar{u}_{k}}{\partial \bar{x}}+\frac{\partial \bar{v}_{k}}{\partial \bar{y}}=0 \text { for } k=1,2 \\
\bar{\rho}_{k}\left(\frac{\partial \bar{u}_{k}}{\partial \bar{t}}+\bar{u}_{k} \frac{\partial \bar{u}_{k}}{\partial \bar{x}}+\bar{v}_{k} \frac{\partial \bar{u}_{k}}{\partial \bar{y}}\right)=-\frac{\partial \bar{p}_{k}}{\partial \bar{x}}+\left(\frac{\partial \bar{\tau}_{x x, k}}{\partial \bar{x}}+\frac{\partial \bar{\tau}_{x y, k}}{\partial \bar{y}}\right) \\
+\bar{\rho}_{k} \bar{g} \sin \beta \text { for } k=1,2, \\
\bar{\rho}_{k}\left(\frac{\partial \bar{v}_{k}}{\partial \bar{t}}+\bar{u}_{k} \frac{\partial \bar{v}_{k}}{\partial \bar{x}}+\bar{v}_{k} \frac{\partial \bar{v}_{k}}{\partial \bar{y}}\right) \\
=-\frac{\partial \bar{p}_{k}}{\partial \bar{y}}+\left(\frac{\partial \bar{\tau}_{x y, k}}{\partial \bar{x}}+\frac{\partial \bar{\tau}_{y y, k}}{\partial \bar{y}}\right)+\bar{\rho}_{k} \bar{g} \cos \beta \text { for } k=1,2,
\end{gathered}
$$

where $\bar{u}_{k}$ and $\bar{v}_{k}$ are, respectively, the $\bar{x}$ and $\bar{y}$ velocity components of layer $k, \bar{p}_{k}$ is the pressure in layer $k, \bar{g}$ is the gravitational acceleration, and $\bar{\tau}_{i j, k}$ is the $(i, j)$ component of the deviatoric stress tensor for layer $k$. The fluids are supposed to be purely viscous, i.e., their viscosity, denoted as $\bar{\eta}_{k}$, depends only on the local shear rate $\bar{\gamma}_{k}=|\partial \bar{u} / \partial \bar{y}|$. They are assumed to follow the four-parameter Carreau model [22]:

$$
\frac{\bar{\eta}_{k}-\bar{\eta}_{\infty, k}}{\bar{\eta}_{0, k}-\bar{\eta}_{\infty, k}}=\left[1+\left(\bar{\delta}_{k} \bar{\gamma}_{k}\right)^{2}\right]^{\frac{n_{k}-1}{2}},
$$

where $\bar{\eta}_{0, k}$ is the viscosity of layer $k$ at low shear rate (Newtonian viscosity limit) and $\bar{\eta}_{\infty, k}$ is the low viscosity limit at high shear rate, $\bar{\delta}_{k}$ is a characteristic time, and $n_{k}$ is a dimensionless parameter. The fluids exhibit a nearly Newtonian behavior both at low and high shear rates, and between these asymptotic trends, the viscosity follows a power-law variation with an exponent $\left(n_{k}-1\right)$.

This Carreau model can be seen as an improvement of the more classic power law, which would not feature this finite viscosity at low shear rate. In the frame of the Carreau model, a fluid is Newtonian when $n_{k}=1$ and shear thinning (or pseudoplastic) when $0<n_{k}<1$ and $\bar{\eta}_{\infty, k}<\bar{\eta}_{0, k}$. A Newtonian behavior can also be obtained by setting $\bar{\delta}_{k}$ equal to zero, whatever the value of $n_{k}$. Note that a shear thickening or so-called dilatant behavior can also be obtained through this model, but this possibility is out of the scope of the present paper.

The base flow denotes the undisturbed flow and is assumed to be parallel and steady. The velocity in each layer is denoted as $\bar{U}_{k}$. For given flow rates per unit width $\bar{Q}_{k}$, the film thicknesses cannot be explicitly calculated and therefore cannot be taken as a length scale, as usually done for Newtonian flows. We adopt the characteristic length scale proposed by Weinstein [18], which is denoted as $\bar{d}_{s}$ and defined by

$$
\bar{d}_{s}=\left(\frac{\bar{\eta}_{0,2} \bar{Q}_{T}}{\bar{\rho}_{2} \bar{g} \sin \beta}\right)^{1 / 3} \text {. }
$$

The dimensionless lengths, velocities, pressure, and time are then chosen as

$$
\begin{aligned}
(x, y) & =\left(\bar{x} / \bar{d}_{s}, \bar{y} / \bar{d}_{s}\right), \quad\left(u_{k}, v_{k}\right)=\left(\bar{u}_{k} \bar{d}_{s} / \bar{Q}_{T}, \bar{v}_{k} \bar{d}_{s} / \bar{Q}_{T}\right), \\
p_{k} & =\frac{\bar{p}_{k} \bar{d}_{s}^{2}}{\bar{\rho}_{2} \bar{Q}_{T}^{2}}, \quad t=\bar{t} \bar{Q}_{T} / \bar{d}_{s}^{2} .
\end{aligned}
$$

The dimensionless flow configuration is shown in Fig. 1.
Dimensionless values have also to be defined for the fluids' properties. We then define density and Newtonian viscosity ratios:

$$
M_{k}=\frac{\bar{\rho}_{k}}{\bar{\rho}_{2}}, \quad E_{k}=\frac{\bar{\eta}_{0, k}}{\bar{\eta}_{0,2}} .
$$

The dimensionless governing parameters are the Reynolds number and the capillary numbers defined as

$$
\operatorname{Re}=\frac{\bar{\rho}_{2} \bar{Q}_{T}}{\bar{\eta}_{0,2}}, \quad \mathrm{Ca}_{k}=\frac{\bar{\sigma}_{k} \bar{d}_{s}}{\bar{\eta}_{0,2} \bar{Q}_{T}} .
$$

Within the framework of the Carreau model (4), the only nonzero dimensionless component of the stress tensor for the base flow can be expressed as

$$
\tau_{x y, k}=\eta_{k} \frac{d U_{k}}{d y},
$$

where $U_{k}=\bar{U}_{k} \bar{d}_{s} / \bar{Q}_{T}$ denotes the dimensionless velocities of the basic flow and

$$
\eta_{k}=E_{k}\left\{I_{k}+\left(1-I_{k}\right)\left[1+\left(L_{k} \frac{d U_{k}}{d y}\right)^{2}\right]^{\left(n_{k}-1\right) / 2}\right\},
$$

with $I_{k}=\bar{\eta}_{\infty, k} / \bar{\eta}_{0, k}$ and $L_{k}=\bar{\delta}_{k} \bar{Q}_{T} / \bar{d}_{s}^{2}$. In the case of a steady parallel flow, Eqs. (2) and (3) become

$$
\begin{gathered}
\frac{d \tau_{x y, k}}{d y}=-M_{k}, \\
\frac{d P_{k}}{d y}=M_{k} \frac{\cot \beta}{\operatorname{Re}},
\end{gathered}
$$

where $P_{k}$ is the dimensionless basic hydrostatic pressure in layer $k$. The no-slip condition at the solid boundary is

$$
U_{2}=0 \quad \text { at } \quad y=d_{1}+d_{2},
$$

where $d_{k}=\bar{d}_{k} / \bar{d}_{s}$ for $k=1,2$ are the dimensionless thicknesses, and the zero shear condition at the free surface is given by

$$
\tau_{x y, 1}=0 \quad \text { at } \quad y=0 .
$$

Additional conditions are specified at the interface between the layers, corresponding to velocity and shear continuity:

$$
\begin{gathered}
U_{1}=U_{2} \quad \text { at } \quad y=d_{1}, \\
\tau_{x y, 1}=\tau_{x y, 2} \quad \text { at } \quad y=d_{1} .
\end{gathered}
$$

The integration of the governing Eqs. (9) and (10) gives

$$
\begin{gathered}
\tau_{x y, k}=-M_{k} y+C_{k} \quad \text { with } \\
C_{1}=0 \quad \text { and } C_{2}=d_{1}\left(1-M_{1}\right), \\
P_{k}=y M_{k} \cot \beta / \operatorname{Re} .
\end{gathered}
$$

By combining Eqs. (7), (8), and (15), we obtain the following differential equations (for $k=1,2$ ):

$$
\begin{aligned}
& E_{k}\left\{I_{k}+\left(1-I_{k}\right)\left[1+\left(L_{k} \frac{d U_{k}}{d y}\right)^{2}\right]^{\left(n_{k}-1\right) / 2}\right\} \frac{d U_{k}}{d y} \\
& \quad=-M_{k} y+C_{k},
\end{aligned}
$$

which have to be solved for $y \in\left[0, d_{1}\right]$ when $k=1$ and for $y \in\left[d_{1}, d_{1}+d_{2}\right]$ when $k=2$, with the boundary conditions 
(11)-(14). A further relation is given by the assumption that the dimensionless total flow rate, $Q_{T}$, is equal to unity:

$$
Q_{T}=Q_{1}+Q_{2}=\int_{0}^{d_{1}} U_{1} d y+\int_{d_{1}}^{d_{1}+d_{2}} U_{2} d y=1 .
$$

Equations (11), (13), (17), and (18) define a nonlinear system which has to be solved to determine the velocity fields $U_{1}(y)$ and $U_{2}(y)$, the shear rates $d U_{1} / d y$ and $d U_{2} / d y$, and the layer thicknesses $d_{1}$ and $d_{2}$. This system cannot be solved analytically in the general case, so that a numerical solution has to be found. A finite difference scheme was used to convert the system of differential equations into a set of algebraic equations. Finally, because of the nonlinearities, an iterative method had to be implemented in order to get the thickness values $d_{1}$ and $d_{2}$ and then deduce the shear rates and basic flow fields.

\section{B. Stability analysis}

To derive the stability problem associated with the basic flow, we use the small perturbation technique. Pressure and velocity components of the disturbed flow can be decomposed as

$$
\begin{aligned}
& u_{k}=U_{k}(y)+u_{k}^{\prime}(x, y, t), \\
& v_{k}=v_{k}^{\prime}(x, y, t), \\
& p_{k}=P_{k}(y)+p_{k}^{\prime}(x, y, t),
\end{aligned}
$$

where a prime is used to denote the small perturbations, and the fluctuation of the interface above the $k$ th layer can be written as

$$
\zeta_{k}=\zeta_{k}(x, t) \text {. }
$$

Neglecting the second-order terms in the perturbation quantities (primed quantities or interface fluctuations), the linearized governing equations for the disturbances are

$$
\begin{gathered}
\frac{\partial u_{k}^{\prime}}{\partial x}+\frac{\partial v_{k}^{\prime}}{\partial y}=0, \\
M_{k} \operatorname{Re}\left(\frac{\partial u_{k}^{\prime}}{\partial t}+U_{k} \frac{\partial u_{k}^{\prime}}{\partial x}+v_{k}^{\prime} \frac{\partial U_{k}}{\partial y}\right) \\
=-\operatorname{Re} \frac{\partial p_{k}^{\prime}}{\partial x}+\frac{\partial \tau_{x x, k}^{\prime}}{\partial x}+\frac{\partial \tau_{x y, k}^{\prime}}{\partial y}, \\
M_{k} \operatorname{Re}\left(\frac{\partial v_{k}^{\prime}}{\partial t}+U_{k} \frac{\partial v_{k}^{\prime}}{\partial x}\right) \\
=-\operatorname{Re} \frac{\partial p_{k}^{\prime}}{\partial y}+\frac{\partial \tau_{x y, k}^{\prime}}{\partial x}+\frac{\partial \tau_{y y, k}^{\prime}}{\partial y},
\end{gathered}
$$

where

$\tau_{x x, k}^{\prime}=2 \eta_{k} \frac{\partial u_{k}^{\prime}}{\partial x}, \quad \tau_{y y, k}^{\prime}=2 \eta_{k} \frac{\partial v_{k}^{\prime}}{\partial y}, \quad \tau_{x y, k}^{\prime}=\theta_{k}\left(\frac{\partial u_{k}^{\prime}}{\partial y}+\frac{\partial v_{k}^{\prime}}{\partial x}\right)$,

and

$$
\begin{aligned}
\theta_{k}= & E_{k}\left\{I_{k}+\left(1-I_{k}\right)\left[1+n_{k}\left(L_{k} \frac{d U_{k}}{d y}\right)^{2}\right]\right. \\
& \left.\times\left[1+\left(L_{k} \frac{d U_{k}}{d y}\right)^{2}\right]^{\left(n_{k}-3\right) / 2}\right\} .
\end{aligned}
$$

Concerning the boundary conditions, they have to be expressed at the perturbed boundaries. However, the linearization and the use of Taylor series expansions allow us to derive conditions which can be applied at the unperturbed boundary locations. At the fixed solid boundary, the no-slip condition is

$$
\begin{gathered}
u_{2}^{\prime}=0 \\
v_{2}^{\prime}=0
\end{gathered} \quad \text { at } \quad y=d_{1}+d_{2}
$$

The kinematic conditions at the free surface and interface are, respectively,

$\frac{\partial \zeta_{k}}{\partial t}+U_{k} \frac{\partial \zeta_{k}}{\partial x}-v_{k}^{\prime}=0$ at $\left\{\begin{array}{lll}y=0 & \text { for } & k=1 \\ y=d_{1} & \text { for } & k=2\end{array}\right.$

At the free surface, the shear stress must vanish, and the normal stress has to balance the surface tension effect. These two conditions are given respectively by

$$
\tau_{x y, 1}^{\prime}-M_{1} \zeta_{1}=0 \quad \text { at } \quad y=0
$$

$p_{1}^{\prime} \operatorname{Re}+\zeta_{1} M_{1} \cot \beta-2 \eta_{1} \frac{\partial v_{1}^{\prime}}{\partial y}-\mathrm{Ca}_{1} \frac{\partial^{2} \zeta_{1}}{\partial x^{2}}=0 \quad$ at $\quad y=0$.

At the interface, both the shear stress and the normal stress induced by each layer have to balance:

$$
\begin{aligned}
& \tau_{x y, 2}^{\prime}-\tau_{x y, 1}^{\prime}-\left(M_{2}-M_{1}\right) \zeta_{2}=0 \quad \text { at } y=d_{1}, \\
& \left(p_{2}^{\prime}-p_{1}^{\prime}\right) \operatorname{Re}+\zeta_{2}\left(M_{2}-M_{1}\right) \cot \beta \\
& +2\left(\eta_{1} \frac{\partial v_{1}^{\prime}}{\partial y}-\eta_{2} \frac{\partial v_{2}^{\prime}}{\partial y}\right)-\mathrm{Ca}_{2} \frac{\partial^{2} \zeta_{2}}{\partial x^{2}}=0 \text { at } y=d_{1},
\end{aligned}
$$

and the continuity of the velocity components must be ensured:

$$
\begin{gathered}
v_{1}^{\prime}=v_{2}^{\prime} \quad \text { at } y=d_{1}, \\
u_{2}^{\prime}-u_{1}^{\prime}=\left(\frac{\partial U_{1}}{\partial y}-\frac{\partial U_{2}}{\partial y}\right) \zeta_{2} \quad \text { at } y=d_{1} .
\end{gathered}
$$

We now assume that there are two-dimensional normal mode solutions of the form

$$
\begin{aligned}
{\left[u_{k}^{\prime}, v_{k}^{\prime}, p_{k}^{\prime}\right](x, y, t) } & =\left[\hat{u}_{k}, \hat{v}_{k}, \hat{p}_{k}\right](y) e^{i(\alpha x-\omega t)} \\
\text { and } \quad \zeta_{k}(x, t) & =\hat{\zeta}_{k} e^{i(\alpha x-\omega t)},
\end{aligned}
$$

where $\alpha$ is the real longitudinal wave number and $\omega$ is the complex frequency. The real part of $\omega, \omega_{R}$, is the angular frequency of the disturbance and the imaginary part, $\omega_{I}$, is the temporal growth rate. The flow is respectively stable, unstable, or neutrally stable according to whether $\omega_{I}$ is negative, positive, or zero. Here $c=\omega / \alpha$ is the complex velocity of the disturbance, and its real part, $c_{R}$, is the phase speed. By substituting these expressions into the linearized perturbation equations (21)(23) and the corresponding boundary conditions (25)-(32), and then eliminating $p_{k}^{\prime}, u_{k}^{\prime}$ and $\zeta_{k}^{\prime}$, we can obtain the linear stability equations for the two-layer flow. They correspond to a system of generalized Orr-Sommerfeld equations for purely viscous 
fluids, with additional terms introduced by the Carreau model:

$$
\left(D^{2}+\alpha^{2}\right)\left[D^{2} \theta_{k}+2 D \theta_{k} D+\theta_{k}\left(D^{2}+\alpha^{2}\right)\right] \hat{v}_{k}-4 \alpha^{2} D\left(\eta_{k} D \hat{v}_{k}\right)=i \alpha \operatorname{Re} M_{k}\left[\left(U_{k}-c\right)\left(D^{2}-\alpha^{2}\right)-D^{2} U_{k}\right] \hat{v}_{k} \quad \text { for } \quad k=1,2,
$$

where $D$ denotes the derivative with respect to $y$. The boundary conditions (25)-(32) become

$$
\begin{gathered}
\begin{array}{c}
D \hat{v}_{2}=0 \\
\hat{v}_{2}=0
\end{array} \text { at } y=d_{1}+d_{2}, \\
{\left[1+\frac{E_{1}}{M_{1}}\left(U_{1}-c\right)\left(D^{2}+\alpha^{2}\right)\right] \hat{v}_{1}=0} \\
i \alpha \operatorname{Re} M_{1}\left(c-U_{1}\right) D \hat{v}_{1}-3 \alpha^{2} E_{1} D \hat{v}_{1}+E_{1} D^{3} \hat{v}_{1}+i \alpha E_{1}\left(\cot \beta+\mathrm{Ca}_{1} \frac{\alpha^{2}}{M_{1}}\right)\left(D^{2}+\alpha^{2}\right) \hat{v}_{1}=0 \quad \text { at } y=0,
\end{gathered}
$$

(note that at the free surface, $y=0$, we have $\theta_{1}=E_{1}$ )

$$
\begin{aligned}
& {\left[\left(1-M_{1}\right)+\theta_{2}\left(U_{2}-c\right)\left(D^{2}+\alpha^{2}\right)\right] \hat{v}_{2}=\theta_{1}\left(U_{2}-c\right)\left(D^{2}+\alpha^{2}\right) \hat{v}_{1}} \\
& \hat{v}_{1}=\hat{v}_{2} \\
& \left(1-M_{1}\right)\left(D \hat{v}_{2}-D \hat{v}_{1}\right)=\left(D U_{1}-D U_{2}\right)\left[\theta_{2}\left(D^{2}+\alpha^{2}\right) \hat{v}_{2}-\theta_{1}\left(D^{2}+\alpha^{2}\right) \hat{v}_{1}\right] \\
& i \alpha \operatorname{Re}\left[\left(c-U_{2}\right) D+D U_{2}\right] \hat{v}_{2}-i \alpha \operatorname{Re} M_{1}\left[\left(c-U_{1}\right) D+D U_{1}\right] \hat{v}_{1}-4 \alpha^{2}\left(\eta_{2} D \hat{v}_{2}-\eta_{1} D \hat{v}_{1}\right) \quad \text { at } \quad y=d_{1} . \\
& \quad \quad+\left(D^{2}+\alpha^{2}\right)\left[D\left(\theta_{2} \hat{v}_{2}-\theta_{1} \hat{v}_{1}\right)+i \alpha\left(\cot \beta+\mathrm{Ca}_{2} \frac{\alpha^{2}}{1-M_{1}}\right)\left(\theta_{2} \hat{v}_{2}-\theta_{1} \hat{v}_{1}\right)\right]=0
\end{aligned}
$$

Equations (34)-(37) correspond to a generalized eigenvalue problem. The numerical procedure used to solve this problem is presented in Sec. III.

\section{NUMERICAL PROCEDURE}

A spectral collocation method based on Chebyshev polynomials is used for the discretization of the Orr-Sommerfeld generalized eigenvalue problem (34)-(37). The system (34)(37) is solved on the Gauss-Lobatto collocation points $\left[y_{j}=\right.$ $\cos (j \pi / N)$ for $j=0, N]$ in each layer. The resulting system of algebraic equations can be written in the abbreviated form

$$
[A] \hat{v}=c[B] \hat{v},
$$

where $\hat{v}$ is the vector containing the algebraic values of the disturbance vertical velocities at each collocation point. The dimension of the square matrices $[A]$ and $[B]$ is twice the number of modes $N+1$. The eigenvalues obtained when solving (38) are the complex angular frequencies $\omega$, and the imaginary part of $\omega$ is the growth rate $\omega_{I}$.

From the spectra obtained by solving (38), we will obtain growth rate curves giving the variation of the maximum growth rates as a function of the wave number. We will also compute neutral curves (values of Re for which an eigenmode has a zero growth rate whereas all the other eigenmodes have a negative growth rate) depending on the wave number $\alpha$, from which critical Reynolds number $\operatorname{Re}_{c}$ can be obtained by minimization along $\alpha$. Note that when different eigenmodes are close to critical in a certain domain of the governing parameters, we will often define a neutral curve and a critical Reynolds number for each of these modes, but it is clear that the true neutral curve and $\mathrm{Re}_{c}$ value will be given by the minimum of these different curves and values.

The numerical procedure has been validated by comparing our results with those obtained by Hu et al. [16] for Newtonian fluids. The agreement concerning the values of the Reynolds number above which instabilities are triggered (neutral curves) is very good.

\section{RESULTS}

We study the stability of a two-layer film flow driven by gravity down an incline when the fluid in the layers have either Newtonian or shear-thinning properties. We will assume that the density of the lower layer is greater than that of the upper layer, i.e., $M_{1}<1$. Without this assumption, the upper layer would become denser than the lower layer, and specific instabilities such as the Rayleigh-Taylor instability could develop. These instabilities will then be out of the scope of this paper. We will particularly analyze how the density and viscosity ratios and the shear-thinning properties of the fluids influence the temporal growth rates of the different free surface and interfacial instabilities.

Four different rheological configurations, denoted with two letters representing the rheology of each layer ( $\mathrm{N}$ for Newtonian and S for shear thinning), will be considered (see Fig. 2).
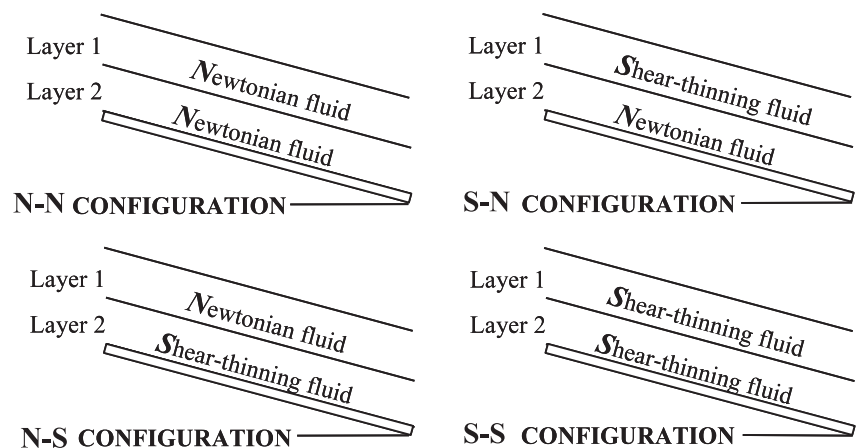

FIG. 2. Sketch of the four rheological configurations studied. 
These configurations are N-N, S-N, N-S, and S-S configurations. The S-N configuration, for example, corresponds to a shear-thinning fluid in the upper layer and a Newtonian fluid in the lower layer. The set of rheological parameters $\left(L_{k} ; n_{k} ; I_{k}\right)$ is chosen equal to $(0 ; 1 ; 0)$ for the Newtonian fluid and $\left(0.8 ; 0.2 ; 10^{-3}\right)$ for the shear-thinning fluid. The inclination of the plane is fixed to $\beta=0.2\left(\beta \approx 11.46^{\circ}\right)$, the same value as in the studies of Chen [14], Loewenherz and Lawrence [2], and $\mathrm{Hu}$ et al. [15,16], the flow rate is fixed to $Q_{1}=0.65$, and the surface tension effects are neglected $\left(\mathrm{Ca}_{1}=\mathrm{Ca}_{2}=0\right)$.

\section{A. Base flow}

When both fluids are Newtonian (N-N configuration), the viscosity is uniform in each layer, so that the system (11), (13), (17), and (18) can be solved analytically. In each layer, the shear rate $d U / d y$ is linear with $y$ with a constant negative slope (remember that $y$ is directed from the free surface to the bottom), and for a given density ratio $M_{1}$, this slope is inversely proportional to the viscosity. This leads, for each layer, to a parabolic base velocity profile, with a curvature inversely proportional to the viscosity. If $E_{1}$ is the viscosity ratio [(upper layer/lower layer)], due to the shear stress continuity at the interface, there is also a change by a factor $1 / E_{1}$ for the absolute shear rate (i.e., equivalently the velocity profile slope) when moving up across the interface.

When the fluid is changed from Newtonian to shear thinning in one of the layers, the viscosity in this layer is expected to decrease, particularly in the zones of high shear rates. Following the observations made in the Newtonian analytic case, this change of viscosity must lead to an increase of the absolute shear rate and of the absolute flow profile curvature. And in the case of a single layer [20], it was found that this change, at constant flow rate, was responsible for a decrease in the layer thickness (connected with an increase of the average velocity) and an increase in the maximum velocity at the free surface. The base flow profiles for the configurations where one of the layers, at least, have a shear-thinning behavior, has to be computed numerically.

\section{Influence of the rheology in a two-layer film for $E_{1}=0.4$}

We first consider the case where the viscosity is smaller in the upper layer $\left(E_{1}=0.4\right)$. The profiles of the viscosity $\eta$, the shear rate $d U / d y$, and the velocity $U$ are plotted as a function of the depth $y$ for the N-N, S-N, and N-S configurations in Fig. 3.

The parameter $E_{1}=0.4$ indicates that, in the Newtonian two-layer configuration ( $\mathrm{N}-\mathrm{N}$ configuration), there is a decrease of the viscosity by this ratio when changing from the lower to the upper layer and a corresponding increase (by $1 / E_{1}=2.5$ ) of the absolute shear rate and of the velocity profile slope at the interface.

In the layers where the fluid is changed from Newtonian to shear thinning, the expected changes in the flow profiles (decrease in viscosity and increase of the absolute shear rate and of the absolute flow profile curvature) are well observed, whereas in the unchanged Newtonian layers, the flow characteristics (viscosity, shear rate, flow profile curvature) are very similar to those obtained in the N-N configuration.

The changes in the shear-thinning layer globally affect the two-layer configuration, with a decrease of the global thickness of the layers and an increase of the velocity at the free-surface. These modifications, however, depend on the position of the shear-thinning layer. If this layer corresponds to the upper layer (S-N case), we have a clear increase of the free surface velocity associated with a small decrease of the global thickness. On the contrary, if this layer corresponds to the lower layer (N-S), the increase of the free surface velocity is moderate while the decrease of the global thickness is significant.

For a better understanding of these results, we can first note that in the S-N case, the flow profile and the thickness of the lower layer are almost unchanged compared with the $\mathrm{N}-\mathrm{N}$ case. The increase of the curvature in the lower part of the upper layer leads to a steeper increase of the velocity in this zone and to an increased free surface velocity. The flow conservation then implies a decrease of the layer thickness, but, due to the high velocities close to the free surface, this decrease can remain moderate. On the other hand, in the N-S case, it is the flow profile in the lower layer which is strongly affected, and this leads to a strong decrease of the lower layer thickness and, as a consequence, of the global thickness.

\section{Influence of the viscosity ratio $E_{1}$}

The viscosity ratio $E_{1}$ is known to play a key role in the stability of superimposed Newtonian fluid films [13]. To

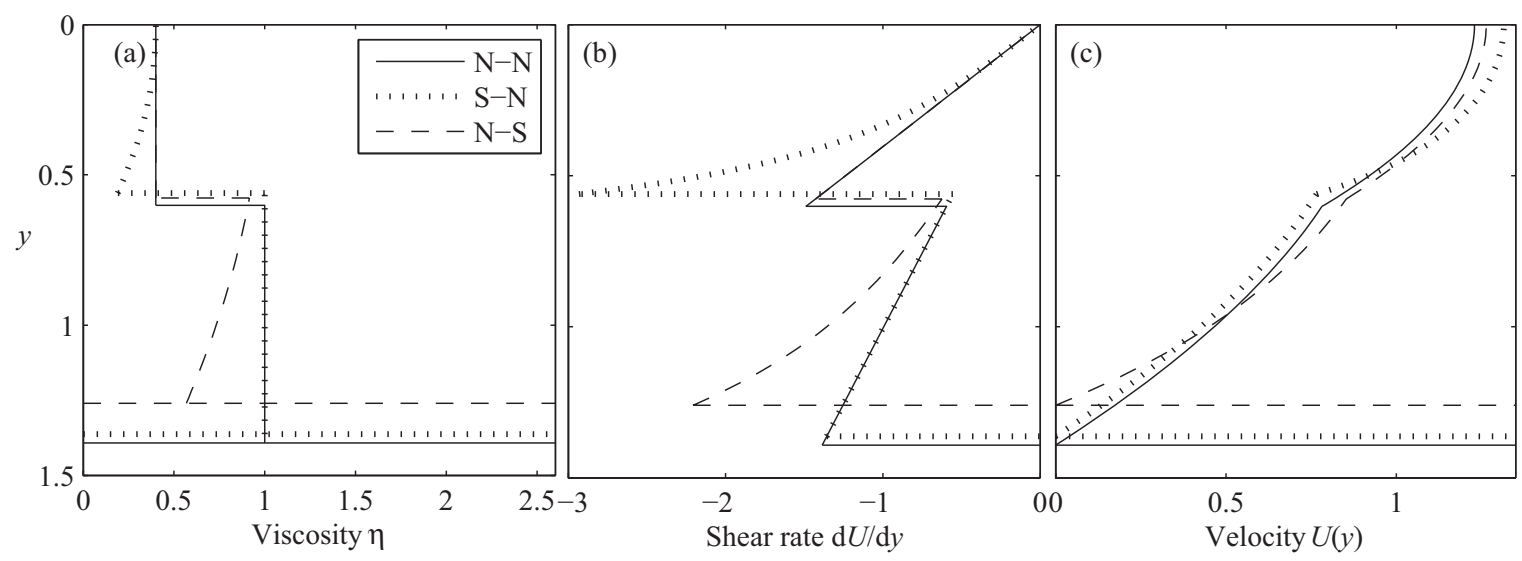

FIG. 3. Base flow viscosity $\eta$ (a), shear rate $d U / d y$ (b) and base velocity profile $U$ (c) as a function of the depth $y$ for a two-layer flow with $E_{1}=0.4, M_{1}=0.99$ and for three different rheological configurations (N-N, S-N, N-S). The other fixed parameters are $\beta=0.2, Q_{1}=0.65$, and $\mathrm{Ca}_{1}=\mathrm{Ca}_{2}=0$. 


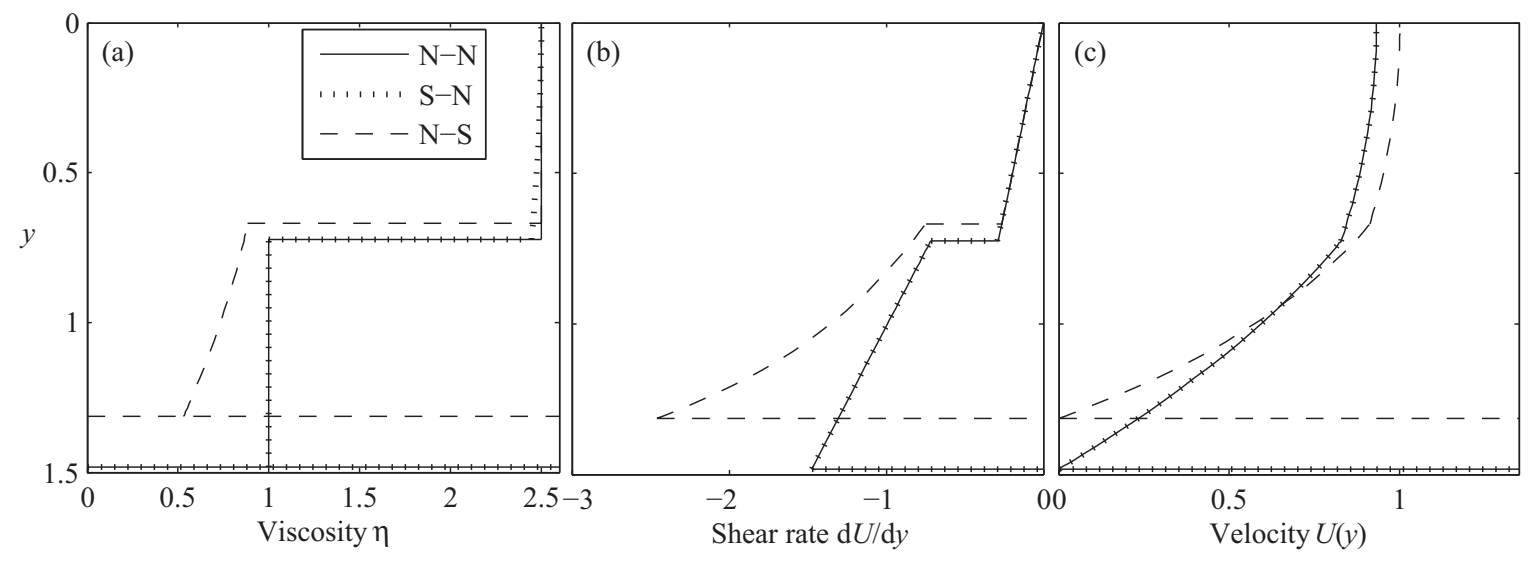

FIG. 4. Base flow viscosity $\eta$ (a), shear rate $d U / d y$ (b), and base velocity profile $U$ (c) as a function of the depth $y$ for a two-layer flow with $E_{1}=2.5, M_{1}=0.99$ and for three different rheological configurations (N-N, S-N, N-S). The other fixed parameters are $\beta=0.2, Q_{1}=0.65$, and $\mathrm{Ca}_{1}=\mathrm{Ca}_{2}=0$.

study the influence of this key parameter on the flow profiles in our configurations, we now consider the case $E_{1}=2.5$ corresponding to a value of the viscosity ratio, which is the inverse of the value used in the previous section. The base flow profiles for this case are shown in Fig. 4 and have to be compared with the results for $E_{1}=0.4$ in Fig. 3 . The change in the viscosity ratio will affect the shear rate ratio between the two sides of the interface, or equivalently the ratio of the velocity profile slopes. In the N-N configuration, these ratios decrease as $1 / E_{1}$, and their value is 0.4 for $E_{1}=2.5$. For example, this will give a larger slope in the upper layer for $E_{1}<1$ (as $E_{1}=0.4$, Fig. 3) and a smaller slope for $E_{1}>1$ (as $E_{1}=2.5$, Fig. 4). In the other configurations, the velocity profile slope ratio at the interface will depend on the local viscosity ratio, which could be different from $E_{1}$.

As shown in Fig. 4, for large values of $E_{1}$ as $E_{1}=2.5$, there is a low shear rate in the upper layer, which results from the stronger viscosity in this zone and the presence of the free surface (zero shear rate at $y=0$ ). As a consequence, the velocity becomes more uniform over the thickness of the upper layer and this thickness increases. Another consequence is that the change from Newtonian to shear thinning in the upper layer fluid has almost no effect on the base flow. The base flow profiles in the N-N and S-N configurations are thus very similar. In contrast, when the change from Newtonian to shear thinning occurs in the lower layer fluid (N-S configuration), the base flow profiles are modified. These modifications (decrease of the global thickness of the layers and increase of the velocity at the free surface), however, are quite similar to those obtained for smaller values of $E_{1}$ as $E_{1}=0.4$ (Fig. 3).

Note finally that when the less viscous fluid becomes shear thinning, i.e., in the $\mathrm{S}-\mathrm{N}$ configuration for $E_{1}<1$ and in the $\mathrm{N}-\mathrm{S}$ configuration for $E_{1}>1$, there is a trend towards the increase of the interface gap between the local values of the shear rates (and hence also of the viscosities). The deviation of the velocity profile through the interface is thus increased, which leads to the thinning of the layer concerned by the change of rheology.

\section{Influence of the density ratio $M_{1}$}

The influence of the density ratio $M_{1}$ is illustrated in Fig. 5 by the plots of the base flow profiles in the S-S configuration for $E_{1}=2.5$ and two different values of the density ratio, $M_{1}=0.85$ and 0.99 .

As shown in Fig. 5, when $M_{1}$ is increased, the free surface velocity, the interface velocity and the shear rate absolute value in both layers increase. Flow conservation then implies that the two layers get thinner. Similar effects are observed for any value of the viscosity ratio $E_{1}$ and for all the different rheological configurations. These effects are slightly accentuated when the fluid in the layers has shear-thinning properties.

\section{B. Linear stability}

The effect of the density ratio $M_{1}$, the viscosity ratio $E_{1}$, and the rheological configuration on the stability of the two-layer flow down an inclined plane is investigated in this section.

Figure 6 shows the surface and interface instability growth rates $\omega_{I}$ as a function of the wave number $\alpha$ in the $\mathrm{N}$ $\mathrm{N}$ configuration, for two values of the viscosity ratio $E_{1}$ [ $E_{1}=0.4$ in Fig. 6(a) and $E_{1}=2.5$ in Fig. 6(b)], different density ratios $M_{1}$, and a low Reynolds number $(\operatorname{Re}=1)$. As shown in previous studies as in $\mathrm{Hu}$ et al. [16], at small values of $\operatorname{Re}$ as $\operatorname{Re}=1$, the surface mode is stable whatever the value of the viscosity ratio $E_{1}$. It is what we observe in Fig. 6 for $E_{1}=0.4$ and 2.5. Stronger values of Re would be necessary to trigger the surface instabilities. Concerning the interface mode, it is found to be stable for $E_{1}=0.4$ in the parameter range studied [Fig. 6(a); the lower layer is the more viscous in this case], whereas Fig. 6(b) for $E_{1}=2.5$ highlights the two unstable wavelengths that can grow for $E_{1}>1$. The long-wave instability growth rate is positive and not much influenced by the density ratio $M_{1}$. In contrast, the short-wave interface mode is stable for small values of $M_{1}$; its growth rate gradually increases when $M_{1}$ is increased, until this mode becomes unstable and eventually supplants the long-wave mode. These results emphasize the existence of limit values of the density ratio, a limit value below which 


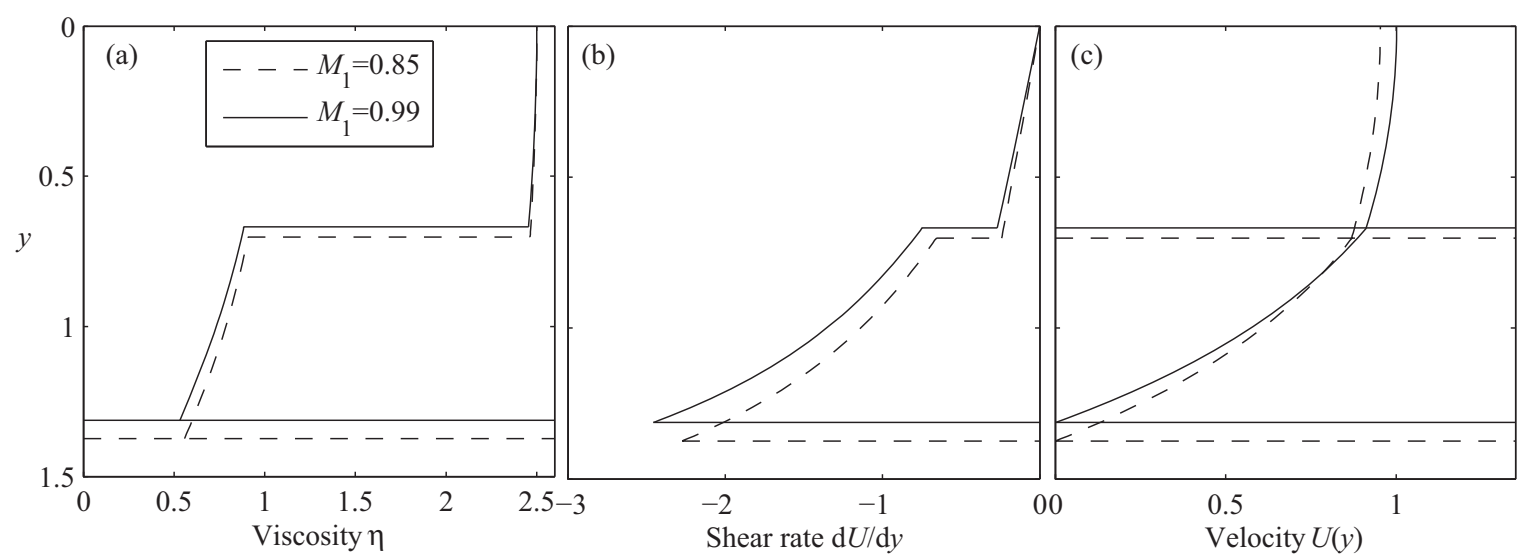

FIG. 5. Base flow viscosity (a), shear rate (b), and base velocity profiles (c) as a function of depth $y$ in the S-S configuration for $E_{1}=2.5$ and for two different density ratios $M_{1}$. The other fixed parameters are $\beta=0.2, Q_{1}=0.65$, and $\mathrm{Ca}_{1}=\mathrm{Ca}_{2}=0$.

the short-wave interface instability disappears, and another limit value above which the interface instability would switch from a long-wave to a short-wave interface mode, as already observed by Hu et al. $[15,16]$. Note that the results concerning the interface mode are in good qualitative agreement with the inertialess results of the literature $[2,9,15]$. The results have also been checked to be in very good quantitative agreement with the results of $\mathrm{Hu}$ et al. [16], which take into account inertia.

For the same governing parameters, we now consider the other rheological configurations involving shear-thinning fluids. Figures 7, 8, and 9 show the instability growth rates as a function of the wave number in the $\mathrm{S}-\mathrm{N}, \mathrm{N}-\mathrm{S}$, and $\mathrm{S}-\mathrm{S}$ configurations, respectively. The curves obtained in these configurations exhibit the same overall variation with $\alpha$ as those obtained in the N-N configuration. Changes, however, can be observed, which can modify the stability properties. For $E_{1}=2.5$, as we have seen in the previous section that the base flow profiles are not influenced by the rheology of the fluid in the upper layer, changes are obtained only in the $\mathrm{N}-\mathrm{S}$ and S-S configurations [Figs. 8(b) and 9(b)], which also give identical results. These changes, however, remain small and mainly affect the interface instability in the small wave number range, $0<\alpha<2$, with a decrease of the growth rate. This induces a slight decrease of the positive growth rate peak at long wavelength, and a stabilization effect in the $\alpha$ range in between the long- and short-wave peaks. The main changes are rather observed for $E_{1}=0.4$. Compared to the case of two Newtonian layers, when the shear-thinning properties are introduced in the upper layer fluid [S-N configuration, Fig. 7(a)], the small and moderate wave numbers $(\alpha \leqslant 4)$ for the interface mode are destabilized, whereas the larger values of $\alpha$ are stabilized. In contrast, when the lower layer rheology is changed [N-S configuration, Fig. 8(a)], there is mainly a stabilization at small wave numbers $(\alpha \leqslant 2)$ for the interface mode and a slight destabilization for larger values of $\alpha$. Finally, for the S-S configuration and the interface mode [Fig. 9(a)], there is mainly a destabilization for moderate wave numbers $(2 \leqslant \alpha \leqslant 4)$. Concerning the surface instability, a slight destabilization is found in the three configurations, but this destabilization is mainly visible in the S-S configuration, with growth rates which become positive.

These results indicate that the density ratio $M_{1}$ as well as the rheological configuration has a significant influence
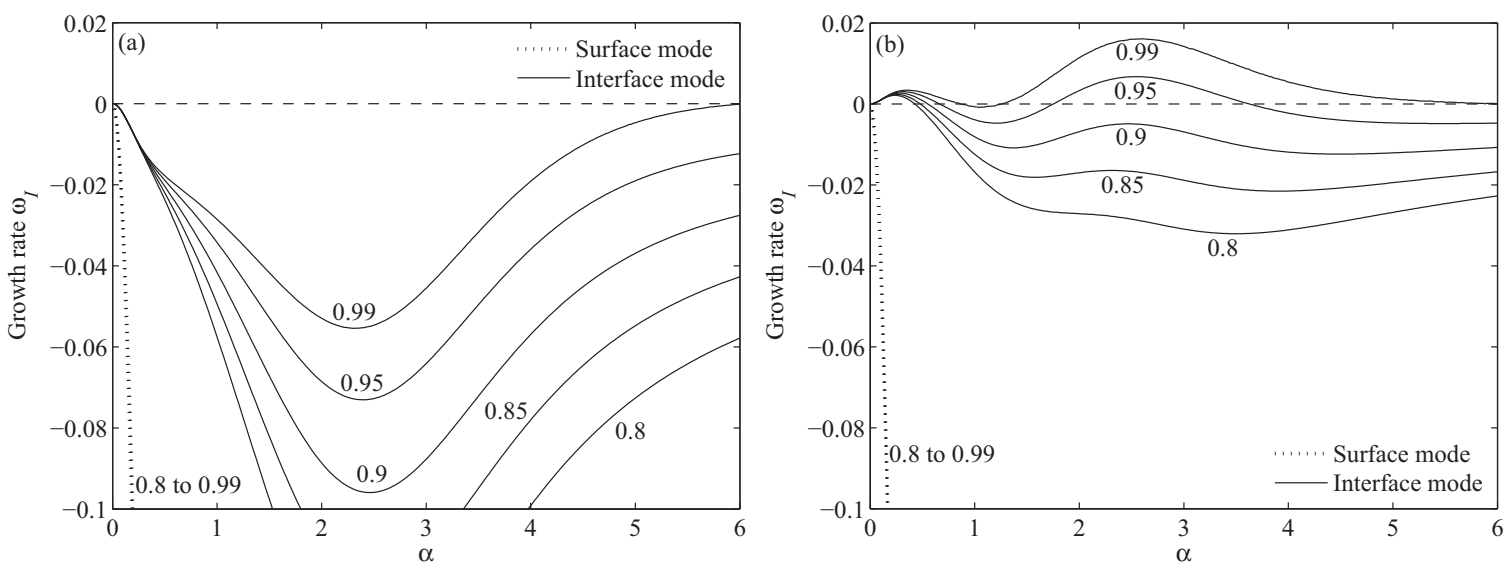

FIG. 6. N-N configuration and $E_{1}=0.4$ (a) or $E_{1}=2.5$ (b): Surface and interface mode growth rate $\omega_{I}$ as a function of the wave number $\alpha$ for $\mathrm{Re}=1$ and for different density ratios $M_{1}$. The other fixed parameters are $\beta=0.2, Q_{1}=0.65$, and $\mathrm{Ca}_{1}=\mathrm{Ca}_{2}=0$. 

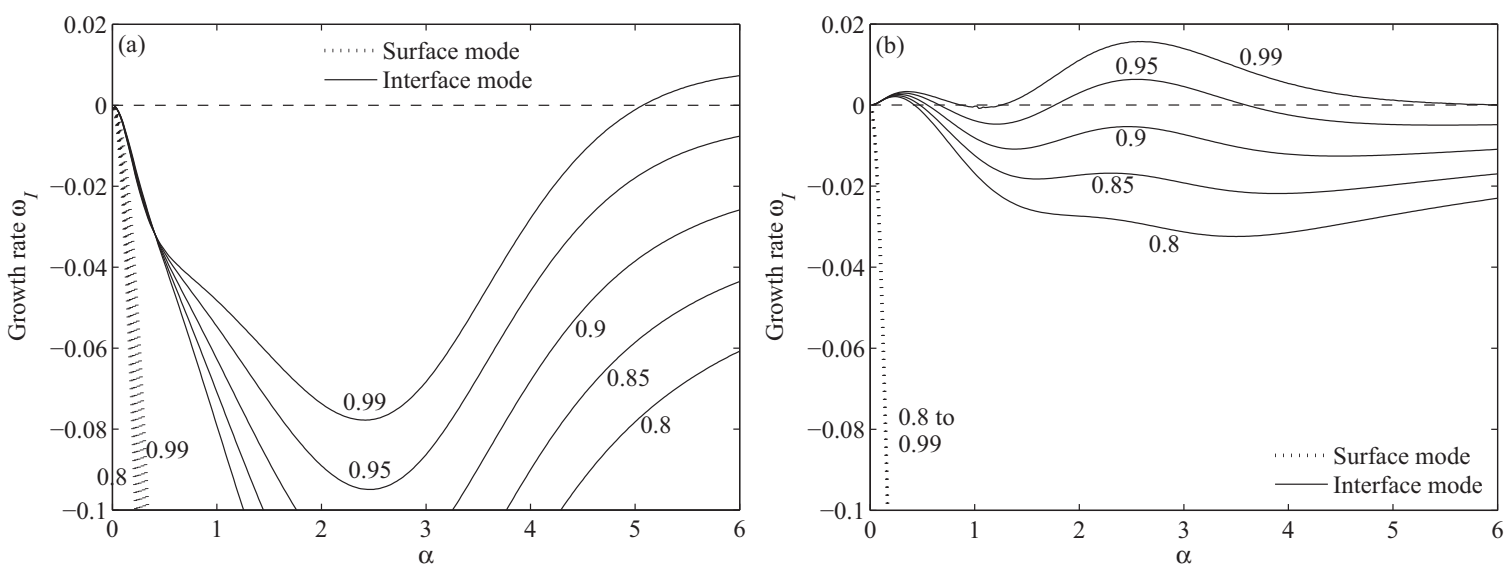

FIG. 7. S-N configuration and $E_{1}=0.4$ (a) or $E_{1}=2.5$ (b): Surface and interface mode growth rate $\omega_{I}$ as a function of the wave number $\alpha$ for $\operatorname{Re}=1$ and for different density ratios $M_{1}$. The other fixed parameters are $\beta=0.2, Q_{1}=0.65$, and $\mathrm{Ca}_{1}=\mathrm{Ca}_{2}=0$.

on the stability of stratified film flows. This influence can be further investigated using stability charts showing the neutral curves as a function of the wave number. Such charts are presented in Figs. 10 and 11 for surface and interface modes in the different rheological configurations. Figure 10 corresponds to the case $E_{1}=0.4$, whereas Fig. 11 corresponds to the case $E_{1}=2.5$. Since the stability results obtained in these two cases are fundamentally different, they will be presented separately in the following. Note finally that we will denote differently the critical Reynolds numbers of each mode (minima of the neutral curves). We will use $\mathrm{Re}_{\text {surf }}$ for the surface mode, $\mathrm{Re}_{l}$ for the long-wave interface mode, and $\mathrm{Re}_{s}$ for the short-wave interface mode. $\mathrm{Re}_{c}$ will be used only to denote a critical Reynolds number in a general way.

\section{Stability charts for $E_{1}=0.4$}

In the case considered in this section, $E_{1}=0.4$, the lower layer is the more viscous. We first comment the results corresponding to the surface modes [Figs. 10(a), 10(c), 10(e), and $10(\mathrm{~g})]$. In all rheological configurations, these modes are stable at low Reynolds number and their destabilization occurs beyond a critical value $\mathrm{Re}_{\text {surf }}$ of $\mathrm{Re}$, at small wave number (long-wave instability). The increase of the density ratio $M_{1}$ is always destabilizing, corresponding to an increase of the unstable zone delimited by the neutral curve, and to a slight decrease of $\mathrm{Re}_{\text {surf. }}$ A similar destabilizing effect is also found, at constant $M_{1}$, when the rheology is changed, i.e., when at least one of the layer is changed from Newtonian to shear thinning. Such influence of the density ratio $M_{1}$ on the surface modes was already observed in the N-N configuration $[13,15,16]$.

Concerning the interface modes, both long- and short-wave instabilities are found, and their neutral curves are connected. In the N-N configuration and for $M_{1}=1$, Chen [14] has shown that the neutral curve delimits a stable region near the origin in the $\operatorname{Re}-\alpha$ plane and then approaches $\operatorname{Re}=0$ when $\alpha \rightarrow \infty$, indicating that the flow is linearly stable for $\mathrm{Re}=0 . \mathrm{Hu}$ et al. [16] later showed that the decrease of $M_{1}$ globally decreases the size of the unstable region (except near $\alpha=0$ ), which indicates that for $M_{1} \leqslant 1$ the flow remains linearly stable for $\mathrm{Re}=0$. Our results show that this behavior is valid for all the rheological configurations [Figs. 10(b), 10(d), 10(f), and $10(\mathrm{~h})]$, so that, in all these cases, inertia is necessary to trigger the interface instabilities.
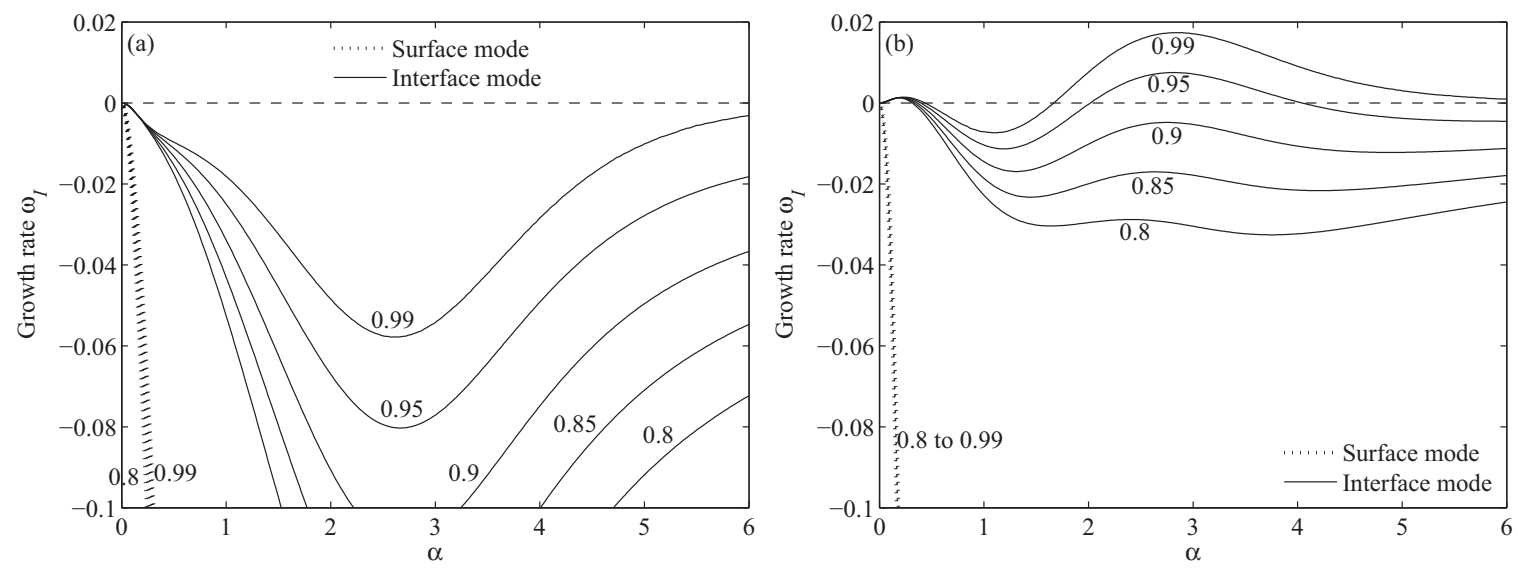

FIG. 8. N-S configuration and $E_{1}=0.4$ (a) or $E_{1}=2.5$ (b): Surface and interface mode growth rate $\omega_{I}$ as a function of the wave number $\alpha$ for $\mathrm{Re}=1$ and for different density ratios $M_{1}$. The other fixed parameters are $\beta=0.2, Q_{1}=0.65$, and $\mathrm{Ca}_{1}=\mathrm{Ca}_{2}=0$. 

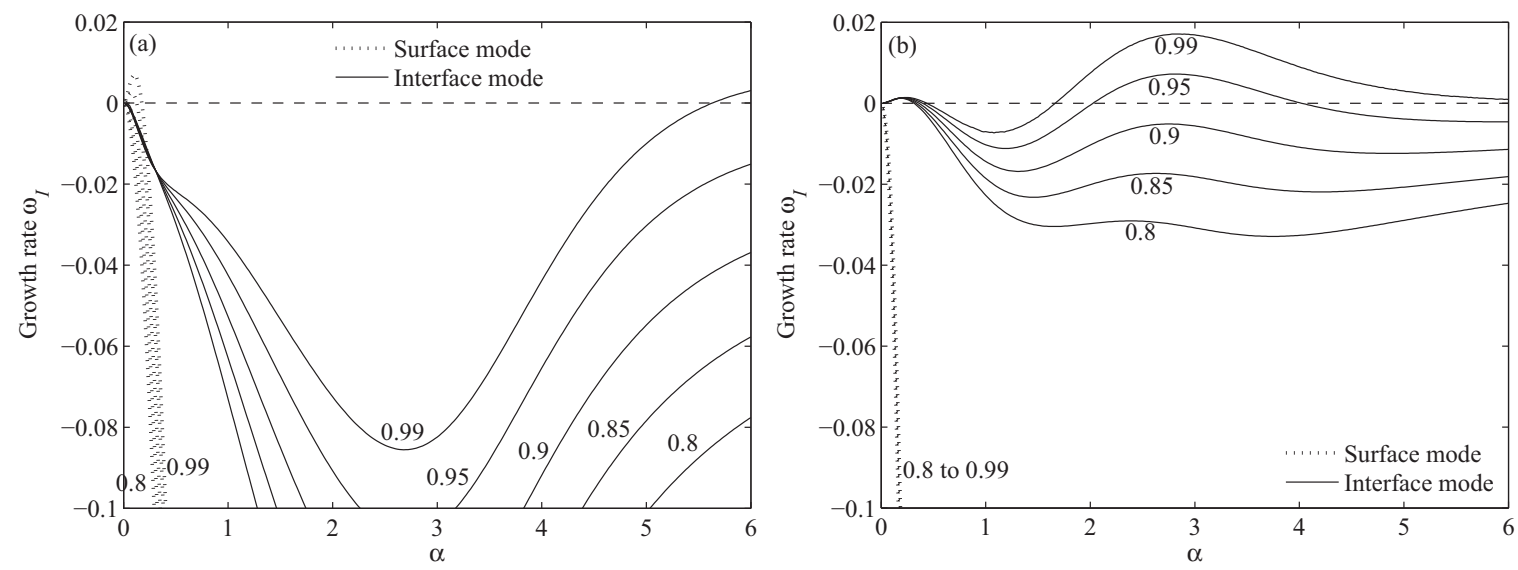

FIG. 9. S-S configuration and $E_{1}=0.4$ (a) or $E_{1}=2.5$ (b): Surface and interface mode growth rate $\omega_{I}$ as a function of the wave number $\alpha$ for $\mathrm{Re}=1$ and for different density ratios $M_{1}$. The other fixed parameters are $\beta=0.2, Q_{1}=0.65$, and $\mathrm{Ca}_{1}=\mathrm{Ca}_{2}=0$.

Figures 10(b), 10(d), 10(f), and 10(h) also give indications on the global changes induced by the change of the rheology in the layers. When the rheology is changed to shear thinning in the lower layer [Fig. 10(f)], we see a destabilization for small wave numbers $(\alpha<2$ or 3$)$ and a stabilization for larger wave numbers. In contrast, when the rheology is changed to shear thinning in the upper layer [Fig. 10(d)], we see a substantial destabilization in the whole wave number range, except for the very small wave numbers $(\alpha \rightarrow 0)$ which are little affected. As a consequence, in the S-S configuration [Fig. 10(h)], the destabilization is still accentuated in the whole wave number range, except the large values of $\alpha$ for which the destabilization is a little less strong than in the S-N configuration. Note that the value of the instability threshold at zero wave number, $\mathrm{Re}_{0}$, is around 20 for both N-N and S-N configurations, whereas it is around 10 for both $\mathrm{N}-\mathrm{S}$ and $\mathrm{S}-\mathrm{S}$ configurations. This indicates that the rheology of the lower layer controls the stability of the system in this very low wave number range. In contrast, the stability at large wave numbers is rather controlled by the rheology of the upper layer.

We now analyze more precisely the influence of $M_{1}$ on the short- and long-wave interface instability for the different rheological configurations:

(1) In the N-N configuration [Fig. 10(b)], the neutral curves intersect at a point [denoted as $\left.\left(\alpha_{\text {cross }}, \mathrm{Re}_{\text {cross }}\right)\right]$ that appears to be independent of the density ratio $M_{1}$. As a result, for $\alpha<\alpha_{\text {cross }}$, the influence of $M_{1}$ on the neutral curves is opposite to the general influence found everywhere else, giving in particular for $\alpha=0$ the stabilizing effect for increasing $M_{1}$ mentionned by Kao [13] in his long wavelength study. To further analyze the results, it is convenient to use the critical values $\operatorname{Re}_{l}$ and $\mathrm{Re}_{s}$ previously defined and corresponding to the minima of the neutral curves: $\mathrm{Re}_{l}$ for the long-wave instability (in the small wave number range) and $\operatorname{Re}_{s}$ for the short-wave instability (in the large wave number range). The analysis of Fig. 10(b) shows that for values of $M_{1}$ close to 1 , the short-wave interface mode is clearly dominant $\left(\operatorname{Re}_{s}<\right.$ $\mathrm{Re}_{l}$ ) and the long-wave interface mode, triggered above $\mathrm{Re}_{l}$, concerns wave numbers which are not very small but rather around 1 . When $M_{1}$ decreases, $\mathrm{Re}_{l}$ increases (stabilization) and the corresponding wave number decreases, until $M_{1} \approx 0.9$, below which this long-wave instability appears for $\alpha \approx 0$ and $\operatorname{Re}_{l}$ decreases (destabilization). The short-wave instability (associated with decreasing $\alpha$ when $M_{1}$ is decreased) remains dominant, however, until $M_{1} \approx 0.83$. Below this value, the long-wave instability (with $\alpha \approx 0$ ) is expected to be dominant.

(2) A similar behavior, with a long-wave instability at $\operatorname{Re}_{l}$ switching from small $\alpha$ to $\alpha=0$, is obtained in the $\mathrm{N}$-S configuration [Fig. 10(f)]. However, due to the changes induced by the change of rheology in the lower layer (destabilization for small wave numbers, stabilization for larger wave numbers), the switching occurs for $M_{1} \approx 0.95$, and the change between short- and long-wave interface mode occurs for $0.9<M_{1}<0.95$.

(3) In the S-N configuration [Fig. 10(d)], the long-wave instability occurs at clearly nonzero values of $\alpha(0.6<\alpha<1)$, which increase with the decrease of $M_{1}$ whereas $\operatorname{Re}_{l}$ increases. The strong destabilization at large wave number in this case allows the short-wave instability to remain the dominant mode in the studied range of $M_{1}\left(0.8 \leqslant M_{1} \leqslant 0.99\right)$.

(4) Finally, the S-S configuration [Fig. 10(h)] is comparable to the previous S-N configuration. The destabilization is, however, stronger at small wave number and weaker at large wave number, so that a transition from the short to the long-wave instability is now observed, which occurs for $0.85<M_{1}<0.9$.

From these observations for the interface instability, we thus see that the limit values of the density ratio $M_{1}$, below which the change of instability from short to long waves takes place, are stronger (closer to 1) when the lower layer fluid becomes shear thinning (N-S and S-S configurations). Note also that the $\mathrm{S}-\mathrm{N}$ configuration is the first configuration to be destabilized at short wavelength (beyond $\mathrm{Re}_{s}$ ) when $\mathrm{Re}$ is increased, whereas the S-S configuration is the first to be destabilized at long wavelength (beyond $\mathrm{Re}_{l}$ ).

We can finally compare the characteristic critical Reynolds number for the surface modes and the interface modes. For values of $M_{1}$ close to 1 , the surface mode and the short-wave interface mode become unstable at values of $\mathrm{Re}, \mathrm{Re}_{\text {surf }}$, and $\mathrm{Re}_{s}$, respectively, which are very close. Depending on the rheological configuration, either one or the other instability will appear first. In contrast, for smaller values of $M_{1}$, the 

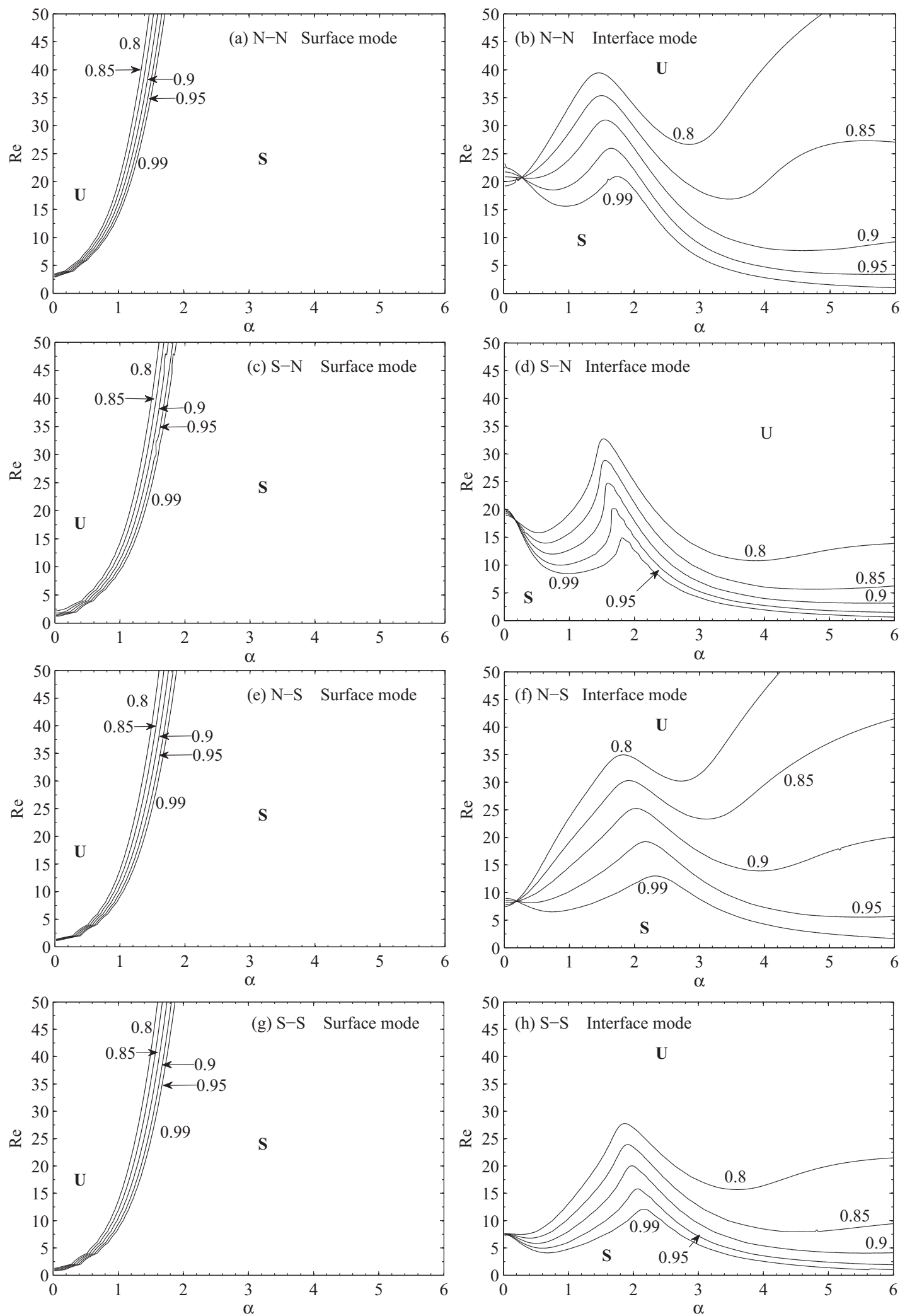

FIG. 10. Stability maps showing neutral curves delimiting stable $(\mathbf{S})$ and unstable $(\mathbf{U})$ areas for different density ratios $M_{1}=$ $0.8,0.85,0.9,0.95,0.99$ and $E_{1}=0.4$ in the different rheological configurations. The other fixed parameters are $\beta=0.2, Q_{1}=0.65$, and $\mathrm{Ca}_{1}=\mathrm{Ca}_{2}=0$.

surface mode will be the first to be unstable, and, depending on the rheological configuration, the long-wave interface mode, triggered at $\mathrm{Re}_{l}$, can even appear before the short-wave interface mode. 

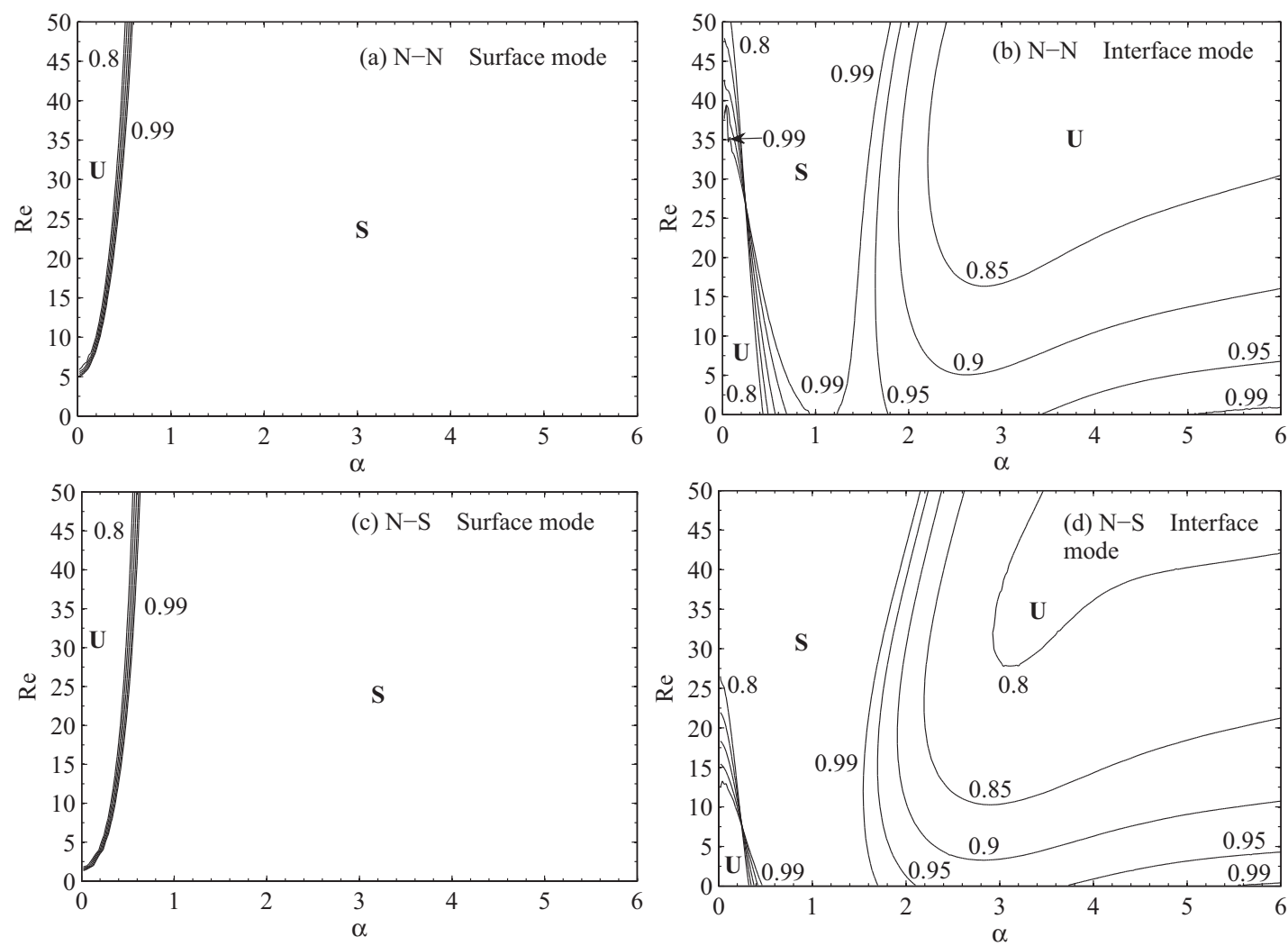

FIG. 11. Stability maps showing neutral curves delimiting stable $(\mathbf{S})$ and unstable $(\mathbf{U})$ areas for different density ratios $M_{1}=$ $0.8,0.85,0.9,0.95,0.99$ and $E_{1}=2.5$ in the N-N and N-S rheological configurations. For this value of $E_{1}$, we verified that the change of the rheology in the upper layer fluid has almost no influence on these stability maps. The other fixed parameters are $\beta=0.2, Q_{1}=0.65$, and $\mathrm{Ca}_{1}=\mathrm{Ca}_{2}=0$.

\section{Stability charts for $E_{1}=2.5$}

In this case we keep the same viscosity ratio between the more viscous and less viscous layers as in the previous section, but the upper layer is now the more viscous $\left(E_{1}=1 / 0.4=\right.$ $2.5)$. The stability maps of the surface and interface modes for this case are presented in Fig. 11. As mentioned in previous sections, for this value of $E_{1}$, the base flow profiles and the instability growth rates are not influenced by the rheology of the fluid in the upper layer. We verified that it is also the case for the stability maps; i.e., the stability maps for the N-N and S-N configurations are almost identical as well as those for the $\mathrm{N}-\mathrm{S}$ and S-S configurations. It is why only the N-N and N-S configurations (corresponding to a change of rheology in the lower layer) are plotted in Fig. 11 and will be discussed in the following.

The surface modes [Figs. 11(a) and 11(c)] are not very sensitive to the viscosity stratification effect, so that the neutral curves for $E_{1}=2.5$ are similar to those obtained for $E_{1}=$ 0.4 [critical value $\mathrm{Re}_{\text {surf }}$ beyond which the surface instability is triggered at long wavelength, destabilizing (though small) influence of the increase of the density ratio $M_{1}$ ]. The change in rheology from Newtonian to shear thinning for the lower layer also induces a decrease of the value of $\mathrm{Re}_{\text {surf }}$, indicating a destabilizing influence.

Concerning the interface modes [Figs. 11(b) and 11(d)], they are unstable in the $\mathrm{Re}-\alpha$ plane in two zones, one zone in the lower left corner below a critical Reynolds number value $\operatorname{Re}_{l, s}$ (the index $s$ denoting a stabilizing limit) (long-wave interface mode) and the other zone in the upper right part of the plane (short-wave interface mode), with an intermediate stable zone centered around $\alpha=1$. As already shown by $\mathrm{Hu}$ et al. [16], there is a clear influence of the density ratio $M_{1}$. For $M_{1}$ close to 1 , the two unstable zones exist at $\operatorname{Re}=0$, and the so-called inertialess instabilities are modified by increasing $\operatorname{Re}$ (stabilization at long wavelength until the disappearance at $\mathrm{Re}_{l, s}$, destabilization at short wavelength). With the decrease of the density ratio $M_{1}$, the long-wave instability is favored as it occurs in a larger range of $\mathrm{Re}$ (increase of $\mathrm{Re}_{l, s}$ ). In fact, the neutral curves for this instability still intersect at a point $\left(\alpha_{\text {cross }}, \operatorname{Re}_{\text {cross }}\right)=(0.3,27)$ which is independent of the density ratio $M_{1}$. As a consequence, for decreasing $M_{1}$, the unstable wave number range and the favored wave number decrease for $\operatorname{Re}<\operatorname{Re}_{\text {cross }}$ whereas they increase for $\mathrm{Re}>\mathrm{Re}_{\text {cross }}$ [see Fig. 6(b)]. In contrast, the unstable region at short wavelength becomes smaller and the instability is eventually triggered only above a critical Reynolds number $\mathrm{Re}_{s}$, which increases as $M_{1}$ is decreased. In some cases, as shown by Hu et al. [16], the unstable region at short wavelength may even disappear at a moderate Reynolds number, below a critical value of the density ratio $M_{1}$.

According to Figs. 11(b) and 11(d), when the lower layer is changed from Newtonian to shear thinning, the long-wave interface mode is stabilized [shrinking of the unstable zone, decrease of the value of $\operatorname{Re}_{l, s}$, change of ( $\left.\alpha_{\text {cross }}, \operatorname{Re}_{\text {cross }}\right)$ to $(0.3$, 
8)] and the short-wave interface mode is destabilized (decrease of the values of $\operatorname{Re}_{s}$ ). We can highlight the consequences of these changes. For example, if we compare Fig. 11(b) with Fig. 11(d) for $\operatorname{Re}=30$, we see that the long wavelengths are always unstable in the N-N configuration, whereas they are stabilized for values of the density ratio $M_{1} \geqslant 0.8$ in the $\mathrm{N}-\mathrm{S}$ configuration. In contrast, at this value of $\mathrm{Re}$ and for $M_{1}=0.8$, the short-wave interface mode is stable in the N-N configuration and unstable in the N-S configuration. In any case, the surface instability is also triggered at this value of Re.

\section{Discussion}

General conclusions can be drawn from the previous analyses. Whatever the viscosity stratification, the surface mode is triggered at large wavelength above a nonzero critical value $\mathrm{Re}_{\text {surf }}$ and is slightly stabilized by the decrease of the density ratio $M_{1}$. Similar trends are found for the short-wave interface mode: this instability is triggered above a critical value $\operatorname{Re}_{s}$ (which could be zero in some cases) and it is stabilized by the decrease of $M_{1}$. In contrast, the viscosity stratification strongly influences the long-wave interface mode, which is unstable below a critical value $\operatorname{Re}_{l, s}=\operatorname{Re}_{0}$ for $E_{1}=2.5$ (inertialess instability) and above a critical value $\operatorname{Re}_{l}$ (which could be $\mathrm{Re}_{0}$ in some cases) for $E_{1}=0.4$. A consequence is that, at long wavelength, the interface mode dominates the surface mode for $E_{1}=2.5$ (i.e., when the upper layer is more viscous) whereas for $E_{1}=0.4$ (i.e., when the lower layer is more viscous), as $\operatorname{Re}_{\text {surf }}<\operatorname{Re}_{l}$, the surface mode is dominant.

Concerning the influence of the rheology, the stability of the short-wave interface mode is rather governed by the rheology of the less viscous layer (upper layer for $E_{1}=0.4$ and lower layer for $E_{1}=2.5$ ), with a destabilizing effect when this layer is changed from Newtonian to shear thinning. In contrast, the stability of the long-wave modes is rather influenced by the rheology of the lower layer, but this influence, depending on the instability concerned and on the value of $E_{1}$, can be stabilizing or destabilizing.

\section{CONCLUSION}

This paper presents a linear stability study of a two-layer film flow down an incline. Three types of instabilities are identified in this configuration: a long-wave surface instability, a long-wave interface instability, and a short-wave interface instability. The fluid in each layer can be considered as Newtonian or shear thinning with a Carreau law behavior. Four configurations are thus possible: N-N, S-N, N-S, and S-S, where, for example, S-N denotes a shear-thinning fluid layer over a Newtonian fluid layer. In each of these configurations, different viscosity and density stratifications can be chosen. In the study, two viscosity ratios [zero-shear viscosity of (upper layer)/(lower layer)] have been considered, $E_{1}=0.4$ and $E_{1}=2.5$, corresponding to cases where the upper layer is, respectively, less and more viscous than the lower layer; and different density ratios $M_{1}$, ranging from 0.8 to 1 and corresponding to a denser lower layer, have been chosen. In contrast, as a first step, the influence of the surface tension (at the upper surface and at the interface) has been neglected.
Note finally that the calculations have been done for a fixed inclination $\beta=0.2$ and a fixed flow rate $Q_{1}=0.65$.

In the general case (layers with shear-thinning properties), the two-layer base flow has to be numerically calculated. The flow rate is imposed and the thicknesses of the layers are computed at the same time as the velocity profiles. The base flow is found to be very sensitive to the viscosity ratio, which induces changes in the velocity profiles slopes and in the thicknesses of the layers. The change of the rheology also affects the base flow. It induces general changes in the velocity profiles, but also important changes in the global thickness of the layers when the lower layer becomes shear thinning. In contrast, the base flow is almost unaffected by a change of the rheology in the upper layer, when this upper layer is the more viscous.

The viscosity stratification greatly influences the stability of the two-layer film flow. When the lower layer is the less viscous (as for $E_{1}=2.5$ ), the interface instability is the more dangerous, since it can grow without inertia. The long-wave interface instability is generally dominant as it has positive growth rates whatever is the value of the density ratio $M_{1}$, but for values of $M_{1}$ close to 1 , the short-wave interface instability can have larger growth rates and become dominant. This well-known inertialess effect no longer exists when the viscosity of the two layers is reversed (as for $E_{1}=4$ ). In this case, the base flow is indeed very different, with different thicknesses of the two layers and different velocity profiles: this affects the velocity perturbation within the layers, giving a lower shear rate at the interface. With such a decrease of the interface shear-stress, the inertialess interface instability is no longer triggered, and the instabilities appear beyond some critical values of the Reynolds number. In this case, the long-wave surface instability is generally the dominant mode, but the short-wave interface instability can become dominant for values of $M_{1}$ close to 1 .

The fluid rheology also has an influence on the stability of the two-layer film flow. This influence is particularly important when the change of rheology concerns the layer with the smallest viscosity.

Indeed, when the near-wall layer is the less viscous, the rheology of this layer completely determines the flow stability, while the rheology of the upper layer has almost no influence on this stability. For a given zero-shear viscosity, increasing the shear-thinning behavior of the near-wall layer leads to a decrease in the growth rates for the long-wave interface mode, whereas it leads to an increase in the growth rates for the two other modes, namely, the long-wave surface mode and the short-wave interface mode. The long-wave interface instability remains always unstable (zero critical Reynolds number) and is still generally the most dangerous instability. The transition to a short-wave interface instability, however, occurs for slightly smaller values of the density ratio $M_{1}$.

When the upper layer is the less viscous, the change of rheology in this upper layer has an influence on the stability of the flow, but it is not the only influence in this case. This change of rheology will principally affect the surface instability and the interface instability in the short wavelength range, with a destabilizing effect on the neutral curves, whereas a change of rheology in the lower layer will destabilize the surface instability and the interface instability in the long wavelength range. 
We have summarized these behaviors by saying that the rheology of the less viscous layer rather governs the stability of the short-wave interface mode, while the rheology of the lower layer rather governs the stability of the long-wave modes.

We can finally note that in place of the Carreau model, a large number of non-Newtonian models could have been studied here (e.g., Eyring, Prandtl, Williamson, De Kee, Powell-Eyring, Sutterby, Yeleswarapu, etc. [22]). All of these models would have lead to the same tendencies as those described in this paper. We have, however, chosen to focus on the Carreau model because it captures very precisely the features of a large amount of fluids. Thus, our paper can be used by an experimenter to predict the flow stability not only qualitatively but also quantitatively. We have shown in particular that the flow stability is dramatically affected by the rheology of the lower layer. The use of the Carreau model will enable the experimenter to fit very precisely the viscosity distribution over the lower layer and to predict the flow behavior very accurately.
[1] S. F. Kistler and P. M. Schweizer, Liquid Film Coating: Scientific Principles and Their Technological Implications (Chapman and Hall, London, 1997).

[2] D. S. Loewenherz and C. J. Lawrence, Phys. Fluids A 1, 1686 (1989).

[3] N. Balmforth, R. Craster, and C. Toniolo, Phys. Fluids 15, 3370 (2003).

[4] C. Ancey, Rhéophysique des pâtes et des suspensions (EDP Science, Paris, France, 1999).

[5] Y. Forterre and O. Pouliquen, J. Fluid Mech. 486, 21 (2003).

[6] T. B. Benjamin, J. Fluid Mech. 2, 554 (1957).

[7] C.-S. Yih, Phys. Fluids 6, 321 (1963).

[8] C.-S. Yih, J. Fluid Mech. 27, 337 (1967).

[9] K. P. Chen, Appl. Mech. Rev. 48, 763 (1995).

[10] A. Hooper and W. Boyd, J. Fluid Mech. 179, 201 (1987).

[11] T. W. Kao, Phys. Fluids 8, 2190 (1965).
[12] T. W. Kao, Phys. Fluids 8, 812 (1965).

[13] T. W. Kao, J. Fluid Mech 33, 562 (1968).

[14] K. P. Chen, Phys. Fluids A 5, 3038 (1993).

[15] J. Hu, S. Millet, V. Botton, H. Ben Hadid, and D. Henry, Phys. Fluids 18, 104101 (2006).

[16] J. Hu, X. Y. Yin, H. Ben Hadid, and D. Henry, Phys. Rev. E 77, 026302 (2008).

[17] W. Y. Jiang, B. T. Helenbrook, and S. P. Lin, Phys. Fluids 16, 652 (2004).

[18] S. J. Weinstein, AIChE J. 36, 1873 (1990).

[19] R. Usha, S. Millet, H. BenHadid, and F. Rousset, Chem. Eng. Sci. 66, 5614 (2011).

[20] F. Rousset, S. Millet, V. Botton, and H. Ben Hadid, J. Fluids Eng. Trans. ASME 129, 913 (2007).

[21] J. Floryan, S. Davis, and R. Kelly, Phys. Fluids 30, 983 (1987).

[22] P. Carreau, D. D. Kee, and M. Daroux, Can. J. Chem. Eng. 57, 135 (1979). 